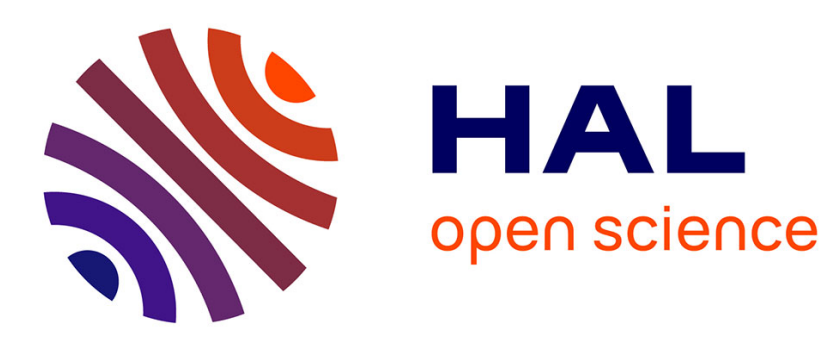

\title{
Electronic Navigational Chart Generator for a marine mobile augmented reality system
}

\author{
Jean-Christophe Morgère, Jean-Philippe Diguet, Johann Laurent
}

\section{To cite this version:}

Jean-Christophe Morgère, Jean-Philippe Diguet, Johann Laurent. Electronic Navigational Chart Generator for a marine mobile augmented reality system. MTS/IEEE OCEANS 2014, Sep 2014, St Jones,

Canada. hal-01083264

\section{HAL Id: hal-01083264 \\ https://hal.science/hal-01083264}

Submitted on 23 Jul 2020

HAL is a multi-disciplinary open access archive for the deposit and dissemination of scientific research documents, whether they are published or not. The documents may come from teaching and research institutions in France or abroad, or from public or private research centers.
L'archive ouverte pluridisciplinaire $\mathbf{H A L}$, est destinée au dépôt et à la diffusion de documents scientifiques de niveau recherche, publiés ou non, émanant des établissements d'enseignement et de recherche français ou étrangers, des laboratoires publics ou privés. 


\section{Electronic Navigational Chart Generator for a Marine Mobile Augmented Reality System}

\author{
Jean-Christophe Morgère \\ Lab-STICC,Université de Bretagne Sud \\ Lorient, France \\ jean-christophe.morgere@univ-ubs.fr
}

\author{
Jean-Philippe Diguet \\ Lab-STICC, CNRS \\ Lorient, France \\ jean-philippe.diguet@univ-ubs.fr
}

\author{
Johann Laurent \\ Lab-STICC, Université de Bretagne Sud \\ Lorient, France \\ johann.laurent@univ-ubs.fr
}

\begin{abstract}
Augmented Reality devices are about to reach mainstream markets but applications have to meet user expectations in terms of usage and ergonomics. In this paper, we present a reallife outdoor Marine Augmented Reality Navigational Assistance Application (MARNAA) that alleviates cognitive load issues (orientation between electronic navigational devices and bridge view) for vessels and recreational boats. First, we describe the current application and explain the requirements to draw relevant and meaningful objects. Secondly we present the 3D chart generator that extracts and provides the meaningful information to the application. Then, we detail our Marine Mobile Augmented Reality System (MMARS) and a generic architecture that can embeds MARNAA application. Finally, we present results and implementations.
\end{abstract}

\section{INTRODUCTION}

To help in marine navigation, there are two kinds of information. The first are related to the seamarks (or landmarks), they are visible from the user's view (bridge view) and indicate some danger or the way to follow. The second are the assistance devices: GPS, AIS, Radar, charts, Electronic Navigational Charts (ENC) that can be in North-up or course-up orientation, which is different from the bridge view. Indeed charts are always printed with the North on the top (North-up) whereas ENC, radar and AIS have often the course of the boat on the top of the display (course-up). This difference between those orientations leads to cognitive issues, the sailor must realize mental arithmetic to put information in the bridge view and to make a decision (to act on the course and boats speed). Moreover in case of emergency, stress increases the probability of making wrong decisions. Porathe [1] and ARVCOP [2] propose solutions that bring together all information from nautical devices such as the bridge view on displays. Their solutions are based on Augmented Reality, so more information than classical marine navigation software or ECDIS (Electronic Chart Display and Information System) are displayed to help the sailor to make the right decision in a very short time. Their applications use the bridge view since the risk of accident is more perceptible in this way. Furthermore, this kind of application can prevent from both collisions and groundings in real time.

To generate all this information to display, a large amount of computation is necessary that's why a PC is needed onboard to run the application. This solution is exclusive to vessels but in marine navigation, recreational boating is also subject to groundings and collision to fixed objects [3]. The only acceptable solution due to ergonomics and low footprint constraints for recreational boats (jet-ski to sailing boats) is a mobile system that can get GPS information, ENC data and display those information in the user's view. First we propose a Marine Augmented Reality Navigation Assistance Application (MARNAA) that is developed on embedded systems with graphical interface under ergonomics, mobile and context (marine navigation) constraints. We take into account a new embedded technology: augmented reality glasses and more precisely a see-through display to let the user see the real world. Second, we introduce a 3D chart generator, which is necessary to handle the tedious conversion of objects. For instance buoys and beacons only represent 128 objects in the entrance of Lorient harbour. For every objects we have to get GPS position, translate it, load the right 3D model in 3D software and export it. We also want flexibility to generate charts depending on the user profile. A recreational, a fishing or a sailing boat don't have the same kind of requirements, for instance regatta data could be introduced with the generator to get race buoys, fishing areas or another example. This generator also reduces onboard computing and offers enough information to get a safe navigation with seamarks, landmarks and danger areas related to the water depth. The generator can be extended to other activity domains such as skiing or hiking. Finally we prototyped a MMARS, which is a fully embedded system that matches with the size and power consumption constraints of recreational boats and professional vessels.

The rest of the paper is organized as follow, Section II is an explanation of the graphical elements chosen in the application that match with ergonomics constraints and maritime context. Section III is a description of the $3 \mathrm{D}$ chart generator that is used to provide charts in accordance with the see-through marine application needs. Our prototype MMARS is described in Section IV and we explain hardware elements required to run MARNAA on a mobile augmented reality system. Then we give results in Section V,finally conclude and draw perspectives.

\section{MARNAA}

\section{A. Maritime context}

On the sea, there are some rules like on the road, there are no signs but navigational information visible on the sea and the land. First, seamarks are compliant with the standard of the International Association of Lighthouse Authorities (IALA) Maritime Buoyage System [4] that defines one system of marks of maritime buoys/beacons. There are specified with four parameters: shape, colour, a top mark and depending on 


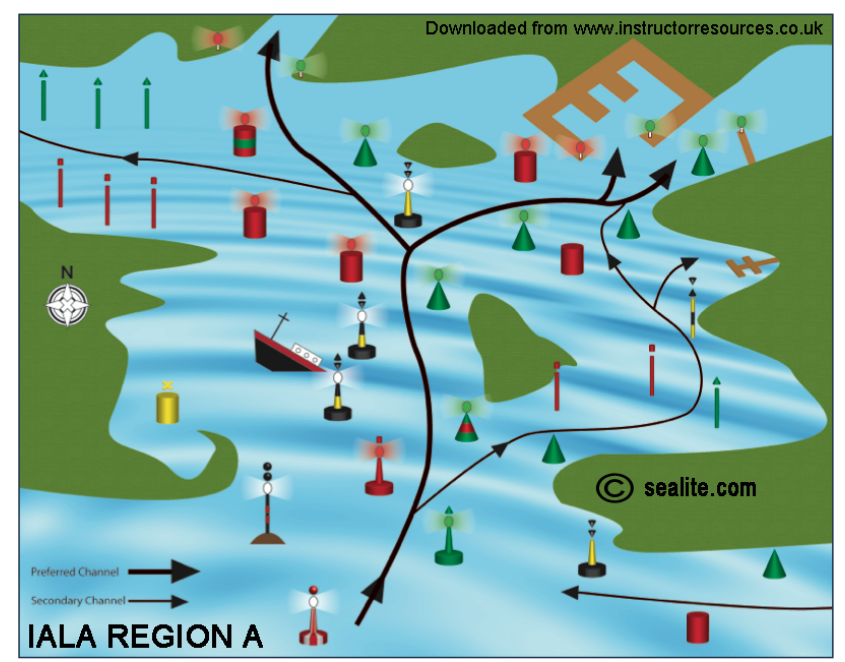

Fig. 1. Representation of an harbour approach with IALA compliance from Sealite.com

the buoy an emitted light that dissociates the type of danger and shows the right way. But for historical reasons, two different systems are used in worldwide: region A and B. Only lateral buoys colour differ from one to the other (all the paper will use region A). Lateral buoys/beacons for instance (red and green buoys Figure 1) define the way to go or to leave the harbour, cardinal buoys/beacons (black and yellow buoys Figure 1) define the way to take, relative to the North, to avoid danger (reef) and isolate danger buoys/beacon (black and red beacon Figure 1) report a danger area around it. Second, the sailor can also used landmarks such as water and church tower, lighthouse to get a fixed point to follow a heading during navigation. All this information (seamarks and landmarks) are specified on the charts or ENC and they are enriched with additional information such as water depths, navigation lines, wrecks (and so on) that are visible on Figure 2. One of the objective of the International Hydrographic Organization (IHO) is to coordinate the activities of national hydrographic offices to uniform nautical charts. For instance S57 [5] is an ENC standard format created by IHO, this is an open source format, so it can be read by marine navigation software or by user's applications. In addition to the charts, some boats have more electronic devices to get a safer navigation. AIS is used to prevent from collision risk with boats as the radar which also detect UFO (Unidentified floating Objects) or reefs in water conditions between calm to moderate. But all those systems (AIS, Radar, charts, ENC and bridge view) increase cognitive load that must be carefully managed.

\section{B. Reduce cognitive workloads}

The use of all those devices is not easy because of the different orientations (Section I) that impact the cognitive load which increases with the number of information sources to decode and the multiple orientation computation. But another factor that could impact the groundings or accidents of the recreational boats is the ignorance or the forgetting of marine navigational rules. This is especially true with occasional use of rental boats. Let's consider the following example with a cardinal buoy. The left part of Figure 3 is a view of nautical

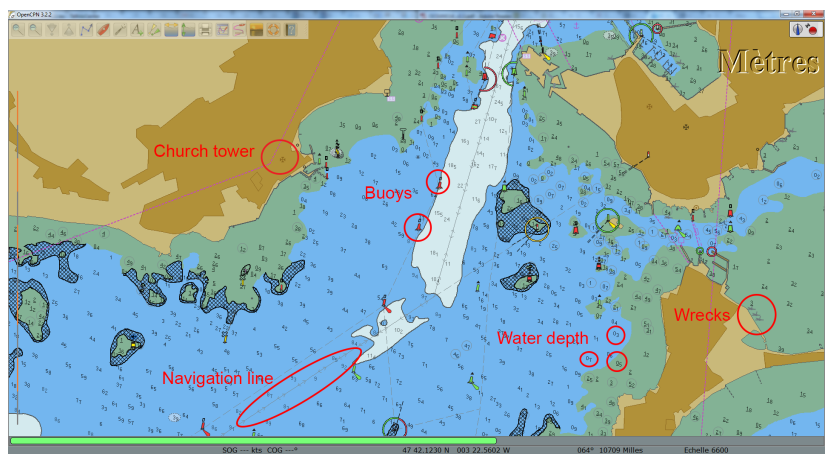

Fig. 2. S57 chart of Lorient harbour loaded with a marine navigation software (OpenCPN)

chart with a North-up orientation. A red symbol represents the boat's position and its course. The user wants to go to the waypoint represented by the yellow mark surrounded with red circle. The sailor looks at the cardinal buoy (right side of Figure 3) and has got a compass that indicates the course of the boat related to the magnetic north. So the sailor has to take the right decision: go on the left (port) or the right (starboard) of the buoy. First the sailor must known the meaning of this buoy (explained Figure 4): the buoy/beacon shows the right way, not the danger position. Second the sailor has to compute orientation between his/her orientation relative to the north (with compass for instance) and the way relative to the north indicated by the buoy to get the right way (see Figure 3). In this example, the sailor has to let the buoy on the port (left) of the boat not on the starboard (right) to avoid the danger, as it is represented by the green and red lines.

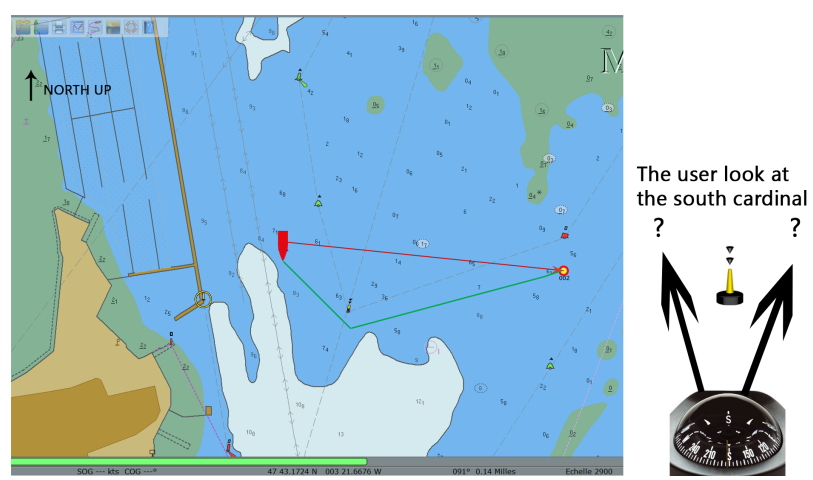

Fig. 3. Example of cognitive load with a cardinal mark

The first solution to reduce cognitive workloads is an application that provides navigational information in the user's view (bridge view) [6]. AIS, Radar data could be displayed on charts depending on the boat's course or heading. In addition to the first solution, the second one is the use of augmented/virtual reality system. In his application, Porathe offers solutions to display danger's positions (reefs, boats, wrecks, ...) with marked area, cone to show far buoys, virtual boats on the display, complex 3D objects, the sea... But our application must be flexible depending on the users needs and habits. A sailor does not need many information with a good visibility, whereas in poor visibility condition he should have more data on the 


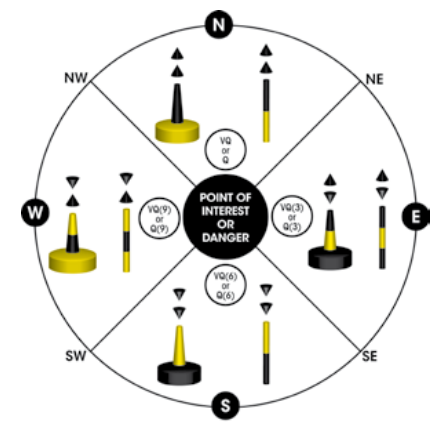

Fig. 4. Cardinal marks meaning with IALA compliance

display. MARNAA runs on portable devices, so we must limit number and complexity of displayed objects and the charts have to be light for memory use. Another key point is the input format of the generator, it must be independent from standard ENC, to let the possibility to customize information by files or load multi vector and raster formats. That's why our application interface is different from existing bridge view applications, it has to be flexible and adapted to the mobile see-through augmented reality context.

\section{Application data}

As introduced in the last paragraph, a mobile augmented reality application must be designed in a different way compared to a classical one. First, a mobile device is limited by computation capacities that impact power consumption and battery life. The second point is the bridge view, we can't display the same information than a marine navigational software because too much information on the display is very tedious to decode and not efficient enough to understand, in a very short time, the boat situation. Third depending on the display technology (see-through, smartphone/tablet) the colours and Field of View will be different than a standard LCD display. We organized our application interface in three categories, which are first the state data related to the GPS, second, the alert data to prevent from risks and third the navigational data with buoys, danger areas and augmented objects.

1) State data: The GPS data are similar to a system status (the boat) with the course, speed, position and the time, it can be related to a supervision system. The user needs course and speed in real time (the most watched), the time should be accessible and the position is only used to correct the course from the next waypoint for instance, so it is useless in a mobile application. We choose the green colour, this colour is preconized because of its high level of lisibility, it is also related to positive connotations [7] (normal state, everything is alright). We have decided to place the boat speed and heading like a list (one below the other) on the Top/Right corner [7] which is a regular seen zone. The time was placed in the Bottom/Right part because this information has to be visible, to check the time if the harbour's entrance conditions depend on the low tide time table for instance. All GPS information were represented by text, the size of characters must also respects rules, the width might be between $50 \%$ to $70 \%$ of the height, with a distance of one meter the characters height has to be
$6 \mathrm{~mm}$ for instance and the font should not be sophisticated [8]. This size depends on the distance and resolution of the mobile display. Even if it limits computations, we added logos to the text, which is more pleasant for the user that was the conclusion of a user inquiry. All data related to the state data are visible Figure 5. The frame is distorted because this is the interface for a specific and distorted AR see-through display and that is why the font is black. There is a scale with numbers that represents the orientation of the device in the bottom of the Figure

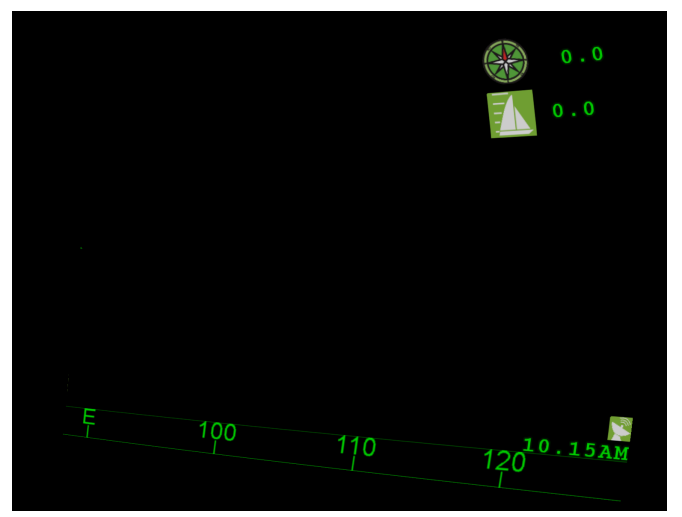

Fig. 5. State data representation in MARNAA

2) Alert data: In general, alerts are located with text or symbols in the up/left zone, this is the most consulted one. The alerts can be a flashing information between 3 and $5 \mathrm{~Hz}$ with a message smaller than 12 characters otherwise there could be some inconveniences [9]. In the case of MARNAA, collision detection alert is displayed with a flashing logo in red and white in case of a collision detection with AIS data, a speed alert can be also added in restricted navigation area such as channel or harbour. A more sophisticated but achievable task could display an alert if the user approaches danger depth areas with grounding risk.

3) Navigation data: For navigation aids, seamarks (buoys, beacons) and daymarks (lighthouse, water tower, church), few models were created and thanks to the augmented reality there are plenty of options to represent them. With ergonomic rules we created 3D seamarks, arrows and symbols. A 3D object is displayed on the buoy's GPS point in accordance with the IALA representation visible Figure 6. There are some constraints due to the see-through technology that imposes system adaptations. First the mobile device is not compatible with IALA rules, indeed some of the buoys (cardinal marks and isolate danger marks) have the black colour. However this is transparency for see-through displays, so it is not possible to see a black object. The solution is the use of gray colour, it is derived from the black colour. Second, AR displays are not enough big, so it is difficult to see an object far from the eye (few kilometers) even if the user have a 3D representation of the seamarks on the display. The best solution are augmented objects: arrows are placed above the buoy to let the information understandable. The arrows have the same colour code as the IALA representation. Third, arrows are not enough readable to decode easily which kind of object is under it. Topmarks can be placed above the arrows to get a better interpretation of the information (Figure 7). Finally, red, yellow and green colour on 
a blue background (sky or sea) constraint the system because this is not the best choice [10] [9], but we have to keep the colours according to user habits and maritime rules, moreover the luminosity can be tuned through the user interface.

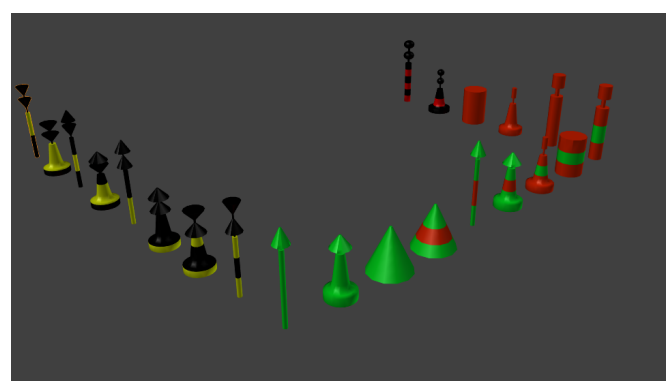

Fig. 6. 3D buoys in accordance with IALA buoyage system

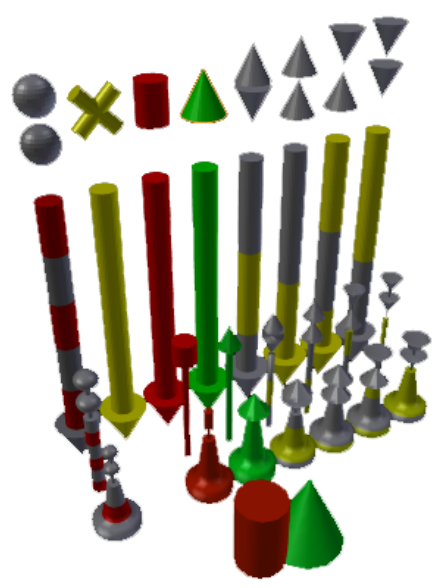

Fig. 7. Mains 3D buoys, arrows and their top marks for see-through display

A cardinal arrow is designed with yellow and grey but the top mark (two cones) could be replaced by the letter that represents the cardinal meaning ( $\mathrm{N}$ for north). This choice must be tested with a large panel because some users want a letter and the other prefer the topmark above the arrow. But the most experienced sailors know the name of the buoys in their sailing area and feel that it could be interesting to get the choice to display the name of the buoys close to the boat. This is not the best choice in terms of ergonomics because some buoys name can be more than fifteen characters that takes a lot of place on the display and could be not efficient enough or negative. Again, the flexibility of the proposed solution make this configuration choice easy to implement and specify in the user profile

The next kind of data is related to the AIS device, we can retrieve boats and prevent collision risk. As the buoys, an arrow is drawn (orange colour) up to the boat position to make it more visible. The last important information are the danger areas depending on the draught of the boat. Actually all the sailors would appreciate to know if their position is safe from groundings or not with an alert or see the edge of the danger. Currently this information is represented with a red area. In ergonomics this is not the best solution, weak density displaying is precognized but with only an edge the user doesn't know if is inside or outside the danger area. Some tests on colours and danger area format will be done with a panel of devices (smartphone, MMARS, AR glasses) to check objects visibility and optimize colours.

All data must be loaded from ENC, so we use a 3D chart generator to place seamarks, landmarks, to generate depth area objects and construct a tree to let the choice for the user to display or not information.

\section{3D NAUTICAL CHART GENERATOR}

\section{A. Input / output}

The generator of the MARNAA application is essential to build 3D nautical charts, because it is very time consuming to read GPS positions, load corresponding objects and place them manually in the $3 \mathrm{D}$ chart. The last task would take many hours even for a small area, such as harbour approaches for example, moreover maps are regulary updated so it is crucial to automate it. The objects, to display in the application, depend on the user profile. Indeed the needs for a recreational boat might be very different from a fishing boat, so we currently conducting surveys to identify those needs. We also need a flexible generator because we must be able to import many formats of input files (vector charts). To validate the whole system we will only use the S-57 format because they are certified by the IHO, which means that they are safer for users, and also that we can get a local chart (Lorient Harbour in this case) to field-test with both the application and 3D charts from the generator.

1) S-57: The Electronic Nautical Chart S-57 is a vectorbased cartographic database, which contains a detailed description of every objects (such as seamarks, shipwrecks, leadlines, sector lights, moorings, etc.); it is the digital equivalent of nautical charts. As they are vector-based and not raster-based, they are more flexible in their use-ways. For instance, you can organize and display information according to a certain area or a navigation mode and also with more accuracy and personalization. It also weighs much less than raster-based maps, around 1 Mo for a map. Furthermore maps are classified in 6 categories according to their scale:

- Cat 1: Global view <1:1500000

- Cat 2: General 1:350000 - 1: 1500000

- Cat 3 : Coast $1: 90000$ - 1:350000

- Cat 4:Approaches 1:22 000 - 1:90000

- Cat 5: Port 1:4000 - 1:22000

- Cat 6 : Mooring > 1:4000

Unlike paper charts, for a geographical area and a given scale, there is only one digital chart. FR602190 for instance is a S57 chart from France with category 6 (Lorient harbour chart). The object's list is defined in the standard and visible in the web site ${ }^{1}$ and it is described as follow: Code, ObjectClass, Acronym, Attribute_A, Attribute_B, Attribute_C, Class and Primitives. An object is an association of two elements: a Feature and a Spatial Object. The Feature Object lists object

${ }^{1}$ http://www.s-57.com/ 
attributes such as the colour, category, size and so on. A Spatial Object is the geographic representation defined by a point, lines or areas.

2) $3 D$ chart: The generator output gives a tree composed of folders containing 3D files with the Wavefront OBJ filename extension and XML files. This tree structure is displayed Figure 8 . The first file generated is metadata.xml (Figure 8) which is a XML file storing the name and the bounding box of the generated maps. It also gives to the application the possibility to load or not the map corresponding to the navigation area of the boat. The last is obtained with the help of the GPS if the user owns the map. The data stored in the application are updated and deals with the loading and unloading of the maps when the boat enters or leaves a zone. The trees folders are named according to the acronyms of the object (from the S-57 standard) in order to be able to sort them out, to write the results and to read them in the MARNAA application. At the bottom of the tree, we can find OBJ files and a XML file (buoy.obj and name.xml for instance), like shown Figure 8. The drawback of the Wavefront OBJ file is that it loses the name of the 3D objects if there are several in the same file. That's why we generate the "name.xml" file that stores object names and their positions. In addition to the two files, the generator adds Wavefront OBJ files, which contained the augmented objects for the navigation application: arrows and topmarks. It is implemented in other files to let flexibility to the user to add or remove those objects in MARNAA.

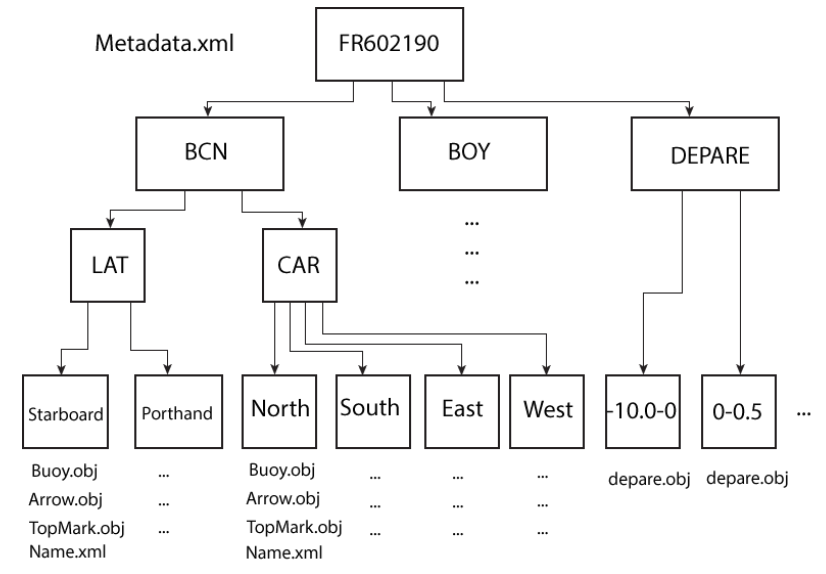

Fig. 8. 3D generator charts output tree

\section{B. Filtering}

1) Sorting: The input filter parameters are the objects to keep in the charts such as buoy, beacon, depth area and so on. To conserve a consistency in all filter steps, the S-57 Acronyms are listed and used as inputs in the generator. Even if the input format file is different from S-57, a conversion will be implemented to keep the use of acronyms in all filter steps. Table I is a data filtering example from a nautical charts (S57). This is a restricted list related to seamarks, landmarks and areas but there are more than 180 different objects defined in the S-57 standard. In this example the generator keeps only some buoys/beacons and depth water area less than 5 meters, which are the essential information for recreational navigation. The depth water area can be adjusted depending on the draught of the boat. Indeed a recreational boat can be a rigid-inflatable boat with a draught of $30 \mathrm{~cm}$ or a sailing boat with 3 meters draught. So this parameter will be asked to the user in the application to adjust the displaying of depth water areas. Some profiles have to be set and use in the generator to complete the 3D charts. The read and the decoding of the data is done with the help of the GDAL (Geospatial Data Abstraction Library), which allows us to translate format geospatial description such as S-57 format. This library provides function to find and store the object's attributes with its acronym as an input. The generator is based on three steps. The first one is the Filtering it has to keep only interesting objects among all. The second is the Writing of information in files and the last one is the building of the 3D charts (Generating).

TABLE I. Selected objects in S-57 ENC

\begin{tabular}{|c|c|c|}
\hline Maritime mark & Meaning & Acronym in S-57 chart \\
\hline \hline Lateral mark & way to go or leave harbour & BCNLAT / BOYLAT \\
\hline Cardinal mark & way to avoid danger & BCNCAR / BOYCAR \\
\hline Isolate danger mark & danger under beacon/buoy & BCNISD / BOYISD \\
\hline Special mark & anchorage areas, pipelines, cables & BCNSPP / BOYSPP \\
\hline Landmark & water tower, church tower,... & LANDMRK \\
\hline Under water rocks & underwater rock, awash rock & UWTROC \\
\hline Depth area & depth area between 0m to 5m & DEPARE \\
\hline
\end{tabular}

2) Universal Transverse Mercator coordinate system: All maps related to the Earth are 2D projections because of the Earth geodesic. It is very difficult to transcribe in a 3D software a surface as the Earth and to use degrees coordinates, so we use in the generator a $2 \mathrm{D}$ projection for the $3 \mathrm{D}$ charts. There are three main kinds of projections. The first one is the equalarea projection, which preserves area measure. The second is the conformal projection which preserves angles locally, so the shapes and finally the equidistant projection which preserves distances between meridians. The 3D chart generator uses the UTM (Universal Transverse Mercator) projection [11], which is a conformal projection. A conformal projection is necessary in navigation because the computing of way are based on angles (marine charts are conformal projection) and the sailors use seamarks or landmarks to follow a course with the help of the compass. On restricted areas (dozen of miles), we consider that the position errors between the $3 \mathrm{D}$ objects seen in the application and the user's view will not be impacted because an UTM projection include some geodetic corrections. However some tests at full-scale will take place in Lorient harbour on a boat with nautical charts from SHOM (National Hydrographic Service) in S-57 format with the category 6 (scale) to identify if the positioning error is acceptable. But this observation can be disturb the GPS error which can vary between 1 to 5 meters and the orientation computing should have an half degree error.

\section{Writing}

In this step, the generator write a $\mathrm{xml}$ file that stores selected objects and information about them. It conserves only attributes related to the shape, position, name and some practical features such as the minimum and maximum depth. The goal is to limit the number of attributes in order to maintain a generic xml file skeleton, which is common to different input formats. The next code is an example of a lateral buoy (type is BOYLAT) that is stored in the xml file. Data are related to the spatial object: the UTM position in the 
tag "point", its UTM zone in the tag "zone". There are also related to the feature object: "Le Pot" (name $=$ Le_Pot) is a port-hand buoy (category $=1$ ) with a cylindrical shape (shape $=2$ ). The category and the shape are enough to retrieve the right $3 \mathrm{D}$ object corresponding to the IALA representation.

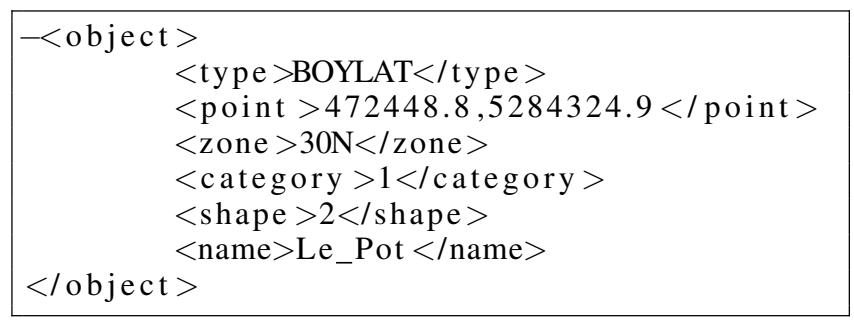

The next code is the $x \mathrm{ml}$ representation of a depth water object (type $=$ DEPARE) which is in the same UTM zone as the lateral buoy but the spatial object is a set of GPS point converted with UTM projection that describes the contour of the area. A depth water object is defined by a minimum and a maximum in the case of a low and high tide. Those values represent the level at the lowest and highest possible astronomical tide, a minimum equals to -7 meters and maximum equals to 0 meter for instance. The generator writes in the xml file all the objects with the same acronym and moves to the next one. This method facilitates the execution of the Generating step.

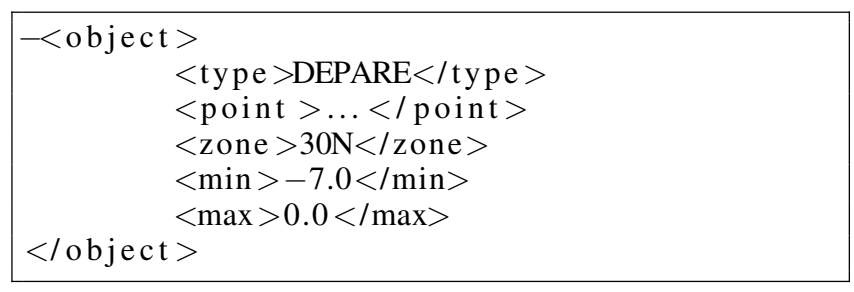

\section{Generating}

The generator depends on the 3D software Blender ${ }^{2}$, some scripts are run with the python api. First, the script read all the same objects stored in the xml file and decode their attributes. If the group of objects is static objects (buoy, beacon, landmark, etc.), the generator looks into the 3D object tree. This one is similar to the output tree and stores all Wavefront OBJ that are necessary to make the 3D charts. When the 3D object matches with its attributes, it is loaded and placed in a 3D scene. When the group is finished, the generator exports the 3D scene as a blender file into the Wavefront OBJ format in the output tree. In addition to the buoys and beacons, augmented objects such as arrows and top marks are also exported and the generator stores the position and name objects in a xml file. When the spatial object is an area, the generator must create a 3D object which represent the contour of this area. This method uses a Bézier curve to create a cylinder all around the area. Today all the area is colored in red as it is shown Figure 9. The left part of the Figure 9 is a representation of an emerged area and the right side is a view of the generated one by the script in blender. We color the area because a simple cylinder is not explicit enough for the user. Indeed, the user

${ }^{2}$ www.blender.org doesn't know if the boat is inside outside of the dangerous area. Then, all the dynamic objects are generated and they are stored in the output tree depending on the depth category: less than 0 meter, $0.0-0.5 \mathrm{~m}, 0.5-1.0 \mathrm{~m}$, etc until 5 meters.
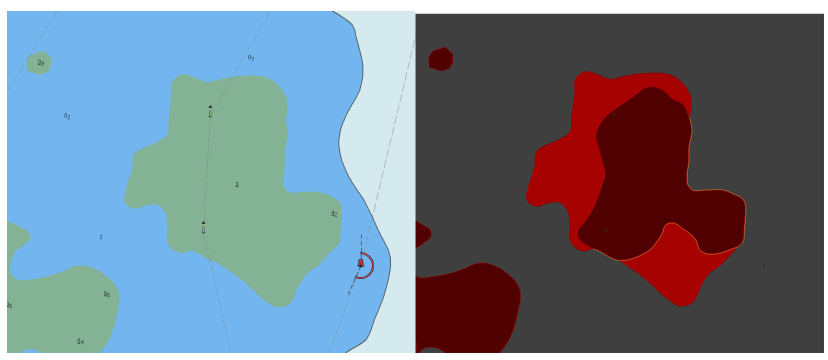

Fig. 9. Navigation software and Blender views of a depth water area

\section{HARDWARE REQUIREMENTS FOR MARNAA} IMPLEMENTATION

\section{A. Marine context}

MARNAA is embedded on a mobile device on a boat, so it involves weather constraints. Indeed the mobile system must provide information under luminosity variations from a shining sea to a dark night. The components that measure and compute orientation must be usable in those conditions but are also constraint by a wide open space, user and boat movements. Swell period vary between $0.05 \mathrm{~Hz}$ and $0.1 \mathrm{~Hz}$ and the frequency required to capture users head movement is $30 \mathrm{~Hz}$. This is the minimum frequency that the system must display information in the right place, in front of the user's eye. In this context, geolocation data are provided by a GPS system because navigational charts are made with a world geodetic system. The system, after geolocation and orientation computing, has to display text, 2D/3D objects to the user, so it needs enough computing capacities to compute and display all information at $30 \mathrm{~Hz}$.

\section{B. Architecture}

1) Orientation: The Microelectromechanical systems (MEMs), in addition with a filter, is one of the best solution for pose estimation in marine navigation because of the boat environment: the weather conditions, the user, the boat movements and the depth field of the scene. We did not choose a camera-based approach, which is not adapted to our context for several reasons. First a camera is disturbed by brightness, which can be very intense on the sea. In case of direct lighting on the lens, the picture cannot be directly exploitable because of the glare. This problem is due to the direct or reflected sunlight on the lens. In addition to the glare, lens flare is caused by a reflection on the lens due to a little angle between the light source and the lens. Some rings or circles may appear on the picture and induce image processing to correct it. Second, in night conditions we could use an infrared camera but unfortunately we would still require a normal camera (like a Smartphone camera) as well that increase the price of the system and introduce more image processing to decode frames extracted from the infrared camera but unfortunately we would still require a normal camera (like a Smartphone camera) as well that increase the 
price of the system and introduce more image processing to decode frames extracted from the infrared camera. Moreover this solution is not adapted to our small footprint and low power objectives. In case of fog, some additional image processing has to be performed to eliminate noise [12], but it requires large computations capacities and far objects might not be perceived anyway. Finally, in normal condition (with no disturbance), some computing must be done on frames such as scale because of the camera FoV (Filed of View), which is different from a real size scene. The use of the camera induces an image processing rate at $30 \mathrm{~Hz}$ and the data flow vary with the camera resolution. For instance, a SVGA resolution has $800 * 600$ pixels coded with 24 bits (in case of RGB) let 190000 Bytes to process every $33 \mathrm{~ms}$ whereas a 720 p resolution are 2764800 Bytes, about the double. In maritime navigation, this is a wide open space, so the camera must acquire small elements in far distance thats why the resolution has to be about $720 \mathrm{p}$ to keep those information. Finally the considered objects are far by definition and the accuracy of the positioning is not critical. So, the incremental cost of image processing compared to the results and our application context dont justify the use of camera in our system.

2) Geolocation: It is possible to have a GPS chip embedded in the system that making it autonomous in this case. It is also possible to connect to a remote GPS with a wireless connection as WiFi or Bluetooth (BT) to get position, course, speed of the boat and signal's accuracy. The application can connect to a RFCOMM server with the BT connection for instance or to an Ad-Hoc network with the WiFi connection.

3) Computing: There are few specific tasks to implement MARNAA. The first one is related to the orientation computing, the system would be more efficient with a Digital Signal Processor onboard or a Floating Point Unit to compute Kalman filters. The second is the geolocation task, when the mobile receives GPS data, it must decode and convert into the right format (UTM projection). But the most important tasks are the graphical ones. Indeed to efficiently compute graphical elements (text, 2D/3D objects) a GPU co-processor is required. It also frees the processor that can handle tasks such as orientation computation, wireless communications and display control. Some graphical APIs are used such as OpenGL ES to compute all 3D specific tasks such as $3 \mathrm{D} / 2 \mathrm{D}$ projection, Z-buffering, lighting, rasterization and frame buffer update.

4) Displays: The mobile system must provide a brightness that is efficient enough to handle luminosity variations from a shining sea (maybe solar-glass) to a dark night. Two kinds of AR devices are available, the smartphones/tablets and AR glasses. The two main characteristics of the displays are the resolution and the Field of View (FoV). First, higher is the resolution and better are the details on the display. So a smartphone/tablet is currently better than AR glasses. Indeed a tablet display have a full HD display or more whereas mobile AR glasses are limited to a Wide VGA $(800 * 600)$ or $1280 \times 720$ resolution with a pocket computer ${ }^{3}$. However our main solution is based on AR glasses since they provide a hand free solution, which is required by sailors, moreover we can expect better resolution in the future. Second, the FoV, for

\footnotetext{
${ }^{3} \mathrm{https}: / /$ www.spaceglasses.com/
}

navigation in a wide open space, should be as large as possible to maximize the space where meaningful data can be displayed and superimposed on the user vision. We have consider two available AR glasses with a FoV of $24^{4}$ and $50^{5}$ in diagonal. It means a very important difference that is precisely illustrated in Figure :fig:FOV with a real picture. One can easily understand that with a FoV of 14 and a low luminosity, the Google glass don't fit with our application requirements. Some arrows can be added to indicate to the user some points of interests are visible out of the FoV. But a too small FoV means a lot of head motions to capture data in a 180 open space and so will be uncomfortable in practice.

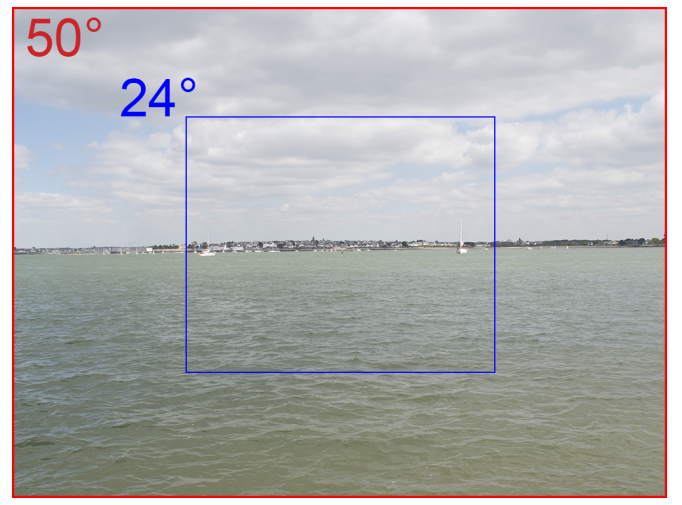

Fig. 10. Difference between two AR glasses Fields of View

\section{IMPLEMENTATION AND RESULTS}

\section{A. MMARS}

1) Orientation: We have implemented on our prototype, an InvenSense IMU (MPU-9150) to acquire data and compute the head orientation with a 9 DOF (Degree of Freedom) device that combines a 3D magnetometer, a 3D accelerometer and 3D gyroscope MEMs. A special task with MEMs is implemented, we want to two kinds of profiles, a low consumption profile and a high accuracy profile to get the choice depending on the needs. So we have implemented the following filters: 7 states EKF (Extended Kalman Filter) [13] and a Gradient Descent algorithm [14]. The first one offers te best racking while the second one is simpler. In addition, MEMs calibrations are required for the magnetometer and the accelerometer due to their offsets and perturbations; it is possible to calibrate those sensors with Kalman filters [15], [16].

2) Geolocation: A wireless communication has been developed to acquire data from the boat network (GPS, AIS, etc.) according to the Bluetooth or WiFi connections. NMEA (National Marine and Electronics Association) ${ }^{6}$ is the most commonly used data format in marine electronics, so GPS, AIS and radar have this data format and consequently easy to parse them. After detecting the header and decode an AIS sentence for instance, we can prevent the user from a collision risk [17] by displaying a warning message.

\footnotetext{
${ }^{4} \mathrm{http} / / /$ optinvent.com/see-through-glasses-ORA

${ }^{5} \mathrm{http}: / / \mathrm{www}$. laster.fr/produits/MG1/

${ }^{6} \mathrm{http}: / /$ www.nmea.org/
} 
3) Computing: MMARS implements a System on Chip (SoC) based on a TI OMAP4430. It includes a dual cortex A9, a PowerVR SGX 540 GPU, a video controller and a power management. Whatever the AR system (AR glasses, MMARS, smartphone or tablets) used, MARNAA application is based on a $3 \mathrm{D}$ engine $^{7}$, which is an open source realtime $3 \mathrm{D}$ engine that can be run on mobile OS such as Android or iOS. The engine uses OpenGL ES2.0 API that allows the programmers to access a lot of 3D functions such as rotate, scale, translate, texturing, lighting and material rendering, according to the vertex and fragment shaders. Of course it requires a GPU.

4) Display: In our experiment setup, the prototype is directly connected to see-through glasses from Laster Technologies shown Figure 11. The model we use is a modified MG1 mask [18], which provides a $800 * 600$ pixels display of 50 degrees of FOV in diagonal and a maximum brightness of $5000 \mathrm{~cd} / \mathrm{m} 2$. This lightness is enough for outdoor applications in shiny environments, whereas mainstream AR glasses are about $3000 \mathrm{~cd} / \mathrm{m} 2$, which is insufficient in such conditions. This system is different to standard Laster products with a limited access, it is dedicated to specific applications that fit with our needs.

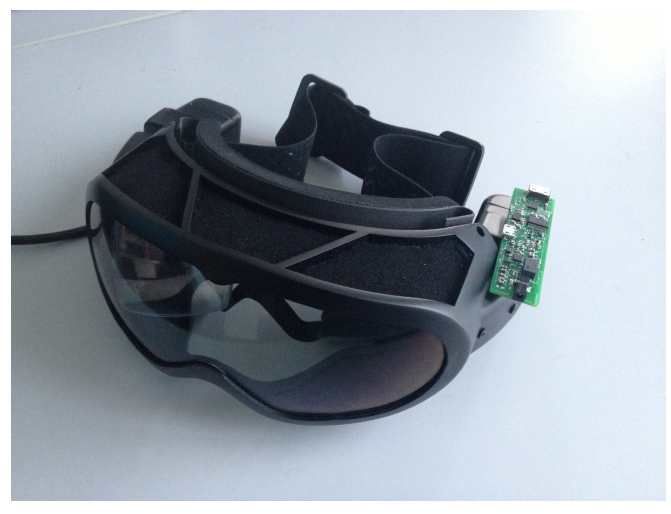

Fig. 11. Marine Mobile Augmented Reality System (first prototype)

\section{B. Standard AR systems}

AR glasses, smartphones and tablets have mobile OS such as Windows, iOS or Android. But the most used in AR glasses is Android, that's why we decided to port the application on Android. The porting of the application on Android is made easier by the fact that both OS share a Linux Kernel. Drivers for the i2C, the GPU or the Wireless connexions are identical and both have OpenGL ES support. However, Android has higher level of abstraction, with managers which allows an easier use of the components but it is less optimised in computations, so it increases the power consumption. For example, the SensorManager can easily control MEMs and get orientation, the LocationManager gets position coordinates, speed, and orientation using the GPS chip and, finally, the Bluetooth adapter can establish a server or a RFCOMM client using the Bluetooth connexion. A native development kit enable us to program in $\mathrm{C}$ and $\mathrm{C}++$ in order to write low-level applications (which have access to the drivers). The graphical part is managed with the free open source 3D engine Irrlicht

${ }^{7}$ http://irrlicht.sourceforge.net/ written in $\mathrm{C}++$. This 3D engine allows the use of OpenGL ES rendering, includes a 2D GUI system and it has been ported on Android. All tasks not related to the hardware such as NMEA decoder, collision detection, MEMs filters are exactly the same on the two systems. But a generic system is not as optimised as a specific one.

\section{C. $3 D$ chart generator}

Currently, the Lorient harbour chart from $\mathrm{SHOM}^{8}$ is used as input file for the 3D generator to facilitate tests in real case. This chart covers a perimeter of 22 nautic miles and takes less than 2 MBytes. The output generated chart with lateral, cardinal, isolate danger, special marks buoys/beacons and all water depth areas less than 5 meters takes less than 150 MBytes. For instance, the memory size of the generated objects and 3D chart of Lorient harbour is visible in the Table II.

TABLE II. MEMORY SIZE OF THE LORIENT HARBOUR 3D CHARTS

\begin{tabular}{|c|c|c|}
\hline Object type & Object number & Memory size (MBytes) \\
\hline \hline Beacon & 82 & 4.6 \\
\hline Buoy & 46 & 1.46 \\
\hline Landmark & 18 & 1.89 \\
\hline Depth area & 537 & 110 \\
\hline
\end{tabular}

The Wavefront OBJ files used for the 3D charts as output files can be loaded easily on the three systems: MMARS, AR glasses and smartphones with the help of the 3D engine. The generator is no dependent from the target or the $3 \mathrm{D}$ engine.

\section{CONCLUSION}

In this paper, we propose a solution for the generation of Electronic Navigational Charts for a complete Marine Mobile AR System. Our generator can convert standard ENC charts (e.g. S-57) into a internal format that is handle by our application (MARNAA). The generator automates the chart building that can depend on user's profile with specific data for recreational, fishing and regatta applications for instance. The whole system (generator, application) has been implemented on our embedded system (MMARS) connected to high-luminosity see-through glasses. It can also be implemented on standard devices such as tablets. We currently considering various improvements mainly related to ergonomics and types of data. We have to make some adjustments on the depth area objects, the chosen color area must be tuned and we have to include the tide of the day, to display more precisely danger areas. Finally, we currently examine the results of a large inquiry that we made to collect feedbacks from different categories of users.

\section{REFERENCES}

[1] T. Porathe, 3-D Nautical Charts and Safe Navigation, ser. Mälardalen University Press dissertations. Mälardalen University, 2006, http://books.google.fr/books?id=NqCcNQAACAAJ.

[2] ARVCOP, http://www.arvcop.com/.

[3] US Coast Guard, Department of Homeland Security (US). 2012 Recreational Boating Statistics http://www.uscgboating.org/assets/1/News/2012ReportR2.pdf.

[4] F. A. Pielou, "The introduction of iala maritime buoyage system a," Journal of Navigation, vol. 31, pp. 422-425, 91978. 
[5] S. IHO, "Transfer standard for digital hydrographic data," S-57, Edition, vol. $3,2000$.

[6] J. Prison and T. Porathe, "Navigation with 2-d and 3-d maps: A comparative study with maritime personnel," Proceedings of the 39th Nordic Ergonomics Society Conference, pp. 1-3, 2007.

[7] M. G. Helander, T. K. Landauer, and P. V. Prabhu, Handbook of HumanComputer Interaction. Elsevier, Aug. 1997.

[8] W. Gilmore, The user- computer interface in process control: A human factors engineering handbook. Elsevier, Dec. 2012.

[9] S. J. Ravden, Evaluating Usability of Human-computer Interfaces: A Practical Method. Ellis Horwood Limited, 1989.

[10] A. Sears and J. A. Jacko, The Human-Computer Interaction Handbook: Fundamentals, Evolving Technologies and Emerging Applications, Third Edition. CRC Press, Sep. 2002.

[11] D. Agency, "The universal grids: Universal transverse mercator (UTM) and universal polar stereographic (UPS)," Defense Mapping Agency, Hydrographic/Topographic Center, Fairfax, VA, USA, Tech. Rep. TM8358.2, 1989.

[12] LYYN, www.lyyn.com.

[13] J. Marins, X. Yun, E. Bachmann, R. Mcghee, and M. Zyda, "An extended kalman filter for quaternion-based orientation estimation using marg sensors," in Intelligent Robots and Systems. IEEE/RSJ International Conference on, vol. 4, 2001, pp. 2003-2011 vol.4.

[14] R. V. Sebastian O.H. Madgwick, Andrew J.L. Harrison, "Estimation of IMU and MARG orientation using a gradient descent algorithm," IEEE International Conference on Rehabilitation Robotics, 2011.

[15] T. Ozyagcilar, "Calibrating an ecompass in the presence of hard and soft-iron interference," Freescale Semiconductor Ltd, 2012.

[16] I. Frosio, F. Pedersini, and N. Alberto Borghese, "Autocalibration of MEMS accelerometers," Instrumentation and Measurement, IEEE Transactions on, vol. 58, no. 6, pp. 2034-2041, June 2009.

[17] L. P. Perera and C. G. Soares, Detections of potential collision situations by relative motions of vessels under parameter uncertainties. Taylor \& Francis Group, London, UK, 2012.

[18] Laster Technologies, http://www.laster.fr/produits/MG1/. 